\title{
Influence of physical rehabilitation on heart rate dynamics in patients with idiopathic pulmonary fibrosis
}

\author{
Sameera Senanayake ${ }^{1,2}$, Nicholas Harrison ${ }^{3}$, Michael Lewis ${ }^{1, *}$ \\ ${ }^{1}$ School of Sport and Exercise Sciences, Swansea University, Swansea, UK \\ ${ }^{2}$ General Sir John Kotelawala Defence University, Rathmalana, Sri Lanka \\ ${ }^{3}$ Respiratory Unit, Morriston Hospital, Abertawe Bro Morgannwg University Health Board, Swansea, UK
}

This study sought to determine whether a 6-week physical rehabilitation programme has a measurable influence on heart rate responsiveness to changing metabolic demand in patients with pulmonary fibrosis. Fifteen individuals ( 13 with idiopathic pulmonary fibrosis and two with pulmonary fibrosis associated with rheumatoid disease) underwent assessments of physical function (6-min walk test), ventilatory function (spirometry), and cardio-respiratory function (respiratory gas analysis and electrocardiogram [ECG] recording during a protocol consisting of periods of rest, incremental bicycle exercise to maximal effort, and post-exercise recovery). RR (beat-to-beat cardiac intervals) data were derived from the ECG and used to quantify (a) heart rate variability (HRV) and (b) cardiac acceleration (AC) and deceleration capacities (DC) (via phase rectified signal averaging). Following the rehabilitation programme, heart rate was elevated by $11 \%-18 \%$ during exercise and re- covery states. HRV was not statistically influenced by rehabilitation during any stage of the assessment protocol; however, qualitative changes were apparent with HRV increasing by $68 \%-75 \%$ during all stages of the protocol. Statistically, $A C$ and DC were similar pre- and postrehabilitation $(A C=-2.7$ and $-3.2 \mathrm{msec} ; D C=2.3$ and $3.2 \mathrm{msec}$, respectively) but again we observed qualitative improvements in these indices of $19 \%$ and $38 \%$, respectively. These results provide initial evidence that physical rehabilitation improves heart rate dynamics (via modulation of autonomic control of heart rate) in idiopathic pulmonary fibrosis patients, even when respiratory and physical functions are unchanged or diminished.

Keywords: Heart rate, Lung disease, Autonomic nervous system, Exercise

\section{INTRODUCTION}

Idiopathic pulmonary fibrosis (IPF) is the commonest of the idiopathic interstitial pneumonias. Whilst its cause is unknown, it results in progressive fibrosis of the alveolar walls and severe impairment of lung function which is usually fatal (Raghu et al., 2015). The histological pattern observed in IPF is described as usual interstitial pneumonia (UIP) and is characterized by excess extracellular matrix within the alveolar interstitium, which typically demonstrates spatial and temporal heterogeneity. Thus, areas of relatively normal lung are interspersed with areas of mild fibrosis, yet other areas show extensive fibrosis with subpleural cysts, termed honeycombing. The latter can be demonstrated using computed tomography scanning of the thorax and is typically worse at the posterior lung bases. The median survival for patients diagnosed with IPF is 2.5 to 3.5 years, although the range is wide (Ley et al., 2011); some follow a slowly declining trajectory over many years whilst others have a rapidly progressive course. Whilst new therapies that slow the decline in lung function have recently been recommended for IPF (Raghu et al., 2015) there is still no cure. Interestingly, the histological and radiological changes of UIP can also be observed in some patients with collagen vascular disease such as rheumatoid disease and this is associated with an increased all-cause mortality (Kelly et al., 2014). Patients with UIP present with symptoms of increasing breathlessness and fatigue which causes them difficulty conducting day-to-day activities and reduces quality of life (Gross and Hunninghake, 2001).

The primary goal of physical exercise-based pulmonary rehabil-
*Corresponding author: Michael Lewis (iD https://orcid.org/0000-0002-6709-9215 School of Sport and Exercise Sciences, Swansea University, Wales, UK E-mail: m.j.lewis@Swansea.ac.uk

Received: September 14, 2018 / Accepted: November 13, 2018
This is an Open Access article distributed under the terms of the Creative Commons Attribution Non-Commercial License (http://creativecommons.org/licenses/by-nc/4.0/) which permits unrestricted non-commercial use, distribution, and reproduction in any medium, provided the original work is properly cited. 
itation (PR) is to "restore patients to the highest possible functional state" (American Thoracic Society, 2000). Whilst PR can improve exercise performance and lung function in other forms of chronic lung disease (such as chronic obstructive pulmonary disease [COPD]) (Spruit et al., 2013), evidence of its influence on IPF patients is limited (Bolton et al., 2013) but growing (Dowman et al., 2017; Tonelli et al., 2017). Dowman et al. (2017) reported improved 6-min walk test distances (6MWD), health-related quality of life (HRQoL) and symptom severity in interstitial lung disease (ILD) patients (including 61 IPF patients) after eight weeks of PR (although these subsequently declined over the following 6 months). Tonelli et al. (2017) also observed substantial improvements in symptom severity, HRQoL and physical function (6MWD and peak power during cycle ergometry) in ILD patients (including $21 \mathrm{IPF)}$ after around 30 rehabilitation sessions. Both of these studies noted that patients with the most severe functional restriction at baseline benefited the most from rehabilitation. Further research is needed to investigate the mechanisms by which these functional improvements are achieved, and it seems reasonable to expect a substantial role of the cardiovascular system in this regard. Whilst it would not be expected that PR would modify the irreversible structural changes of fibrotic lungs, it is reasonable to consider that it might influence the cardiovascular system. We previously showed that IPF patients' peak $\mathrm{VO}_{2}$ values were half those of similarly aged healthy individuals, whilst their peak heart rates (HRs) were 20\% lower and their kinetic HR responses to heavy intensity exercise were 2.5 times slower (McNarry et al., 2017). These results provide evidence of reduced tissue oxygen utilization following the onset of exercise in people with UIP, which in large part results from impeded central (cardiovascular) oxygen delivery. It would therefore be useful to determine whether a physical rehabilitation programme could improve systemic oxygen delivery (and peripheral muscle oxygen availability) in people with UIP.

HR dynamics are thought to provide a good proxy for the response of cardiac output and muscle blood flow to increased metabolic demand (MacPhee et al., 2005; Yoshida and Whipp, 1994), albeit they do not account for the contribution of stroke volume in meeting this demand. One way to determine HR responsiveness is to measure the variation in beat-to-beat duration of the cardiac cycle or HRV (Lewis and Short, 2010). This technique has been widely used to estimate the influences of the autonomic nervous system (ANS) on HR in various populations. However, caution must be applied when HRV is used to determine an individual's capacity to increase or decrease HR. Firstly, because HRV must be determined during steady-state conditions and for extended periods. Hence, it cannot provide an overall measure of the range of cardiac dynamics that occur across multiple states or that are transient in nature. Secondly, the use of HRV as an independent measure of sympathetic and parasympathetic control is not straightforward (Lewis and Short, 2010). An alternative measure of cardiac dynamics that avoids these restrictions is the use of phase-rectified signal averaging (PRSA) (Bauer et al., 2006). This method yields averaged beat-to-beat cardiac intervals (RR) time series that separately characterize all HR modulations that result in accelerations and decelerations.

It is known that individuals with COPD have a degree of cardiac autonomic dysfunction, reflected by altered HR variability (HRV) during rest and exercise (Bartels et al., 2003) but with a normal diurnal variation (Lewis et al., 2009). They also have a tendency towards reduced PRSA-derived cardiac dynamic capacities that are ameliorated with oxygen therapy (Lewis et al., 2011). However, as far as we are aware, neither HRV nor cardiac acceleration/deceleration capacities have been evaluated in individuals with UIP. A comparison of these variables before and after physical rehabilitation might indicate whether they would benefit from such interventions in terms of improved cardiac responsiveness to everyday activities.

In this context, the aim of this study was to determine the influence of an 8-week, exercise-based PR programme on measures of cardiac autonomic control in patients with UIP. Specifically, we sought to quantify HRV and indices of cardiac acceleration and deceleration capacity for these individuals during periods of rest and exercise.

\section{MATERIALS AND METHODS}

\section{Participants}

The study was performed in accordance with the Declaration of Helsinki. Ethical approval to conduct the study was obtained (approval number: 16/WA/0272) from Wales Research Ethics Committee 6, part of the UK Health Departments' Research Ethics Service. Approval and permission to execute the study was obtained from the local health board (Abertawe Bro Morgannwg University Health Board). Recruitment was led by a consultant chest physician, who informed eligible patients about the study (including its purpose, protocol, expected commitment, and the possible risks of involvement). Patients attending the Interstitial Lung Disease Clinic at Moriston Hospital, Swansea, who had been diagnosed with UIP (either IPF or UIP associated with rheuma- 
Table 1. Patient demographics

\begin{tabular}{lccccc}
\hline Patient ID & Age $(\mathrm{yr})$ & Gender & $\mathrm{BMl}\left(\mathrm{kg} / \mathrm{m}^{2}\right)$ & $\mathrm{FEV}_{1,} \mathrm{~L}(\%)$ & $\mathrm{FVC}, \mathrm{L}(\%)$ \\
\hline P01 & 72 & $\mathrm{M}$ & 36.7 & $1.92(67)$ & $2.33(62)$ \\
P02 & 73 & $\mathrm{~F}$ & 47.7 & $1.46(82)$ & $1.66(77)$ \\
P03 & 74 & $\mathrm{M}$ & 26.3 & $2.34(78)$ & $2.78(63)$ \\
P04 & 67 & $\mathrm{~F}$ & 34 & $1.48(75)$ & $1.81(76)$ \\
P05 & 78 & $\mathrm{M}$ & 32.7 & $2.93(130)$ & $3.95(131)$ \\
P06 & 60 & $\mathrm{~F}$ & 32 & $2.73(88)$ & $3.49(89)$ \\
P07 & 68 & $\mathrm{M}$ & 32.3 & $2.53(79)$ & $3.22(74)$ \\
P08 & 83 & $\mathrm{M}$ & 28.7 & $2.21(85)$ & $2.90(82)$ \\
P09 & 73 & $\mathrm{M}$ & 23.1 & $1.93(63)$ & $3.13(52)$ \\
P10 & 75 & $\mathrm{M}$ & 20.8 & $2.22(93)$ & $3.37(107)$ \\
P11 & 68 & $\mathrm{~F}$ & 30.4 & $1.77(60)$ & $2.12(53)$ \\
P12 & 72 & $\mathrm{M}$ & 25.3 & $2.00(63)$ & $1.46(61)$ \\
P13 & 64 & $\mathrm{~F}$ & 30.1 & $1.12(55)$ & $1.46(59)$ \\
P14 & 65 & $\mathrm{M}$ & 24.5 & $2.50(78)$ & $3.25(78)$ \\
P15 & 55 & $\mathrm{M}$ & 29.8 & $\mathrm{NA}$ & $\mathrm{NA}$ \\
\hline
\end{tabular}

$\mathrm{BMI}$, body mass index; $\mathrm{FEV}_{1}$, age-predicted forced expiratory volume in $1 \mathrm{sec}$; FVC, age-predicted forced expiratory volume; NA, not available.

toid disease) by a Multi-Disciplinary Team, according to the American Thoracic Society (ATS) and European Respiratory Society (ERS) guidelines (Raghu et al., 2011) were eligible to participate. All recruited individuals provided their written informed consent to participate in the study. Eighteen individuals (13 male, 5 female) agreed to participate in the study but three males later withdrew from the rehabilitation programme for personal reasons. Patients continued with their usual medication regimes whilst participating in the study. The patients' demographics are shown in Table 1.

\section{Exclusion criteria}

- Respiratory infection within the previous 2 months.

- Known coronary or valvular heart disease.

- Any other serious comorbid condition (e.g., emphysema, uncontrolled diabetes, renal disease) Significant hypoxia (oxygen saturation below 85\%) when breathing air and undergoing conventional screening for a PR programme.

- Musculoskeletal problems that would make it impossible to exercise.

- Current smokers.

\section{Experimental protocol}

Participants were asked to visit the lung function laboratory at Morriston Hospital, Swansea, for baseline physiological assessment. They were also asked to not consume alcohol for $24 \mathrm{hr}$ before each test day, to not consume caffeine for $6 \mathrm{hr}$ prior to the scheduled tests, but otherwise to have eaten and consumed fluids as normal. All tests were conducted on the same time of the day $\pm 1 \mathrm{hr}$.

Participants undertook a 6-min walk test to determine their functional (walking) capacity (6MWD), in line with ATS guidelines (ATS Committee on Proficiency Standards for Clinical Pulmonary Function Laboratories, 2002). On a separate day they were asked to complete an incremental exercise test on a stationary bicycle (ergometer) (VIAsprint150P Ergometer, Vyaire Medical, Mettawa, IL, USA) in accordance with methods described by Arena and Sietsema (2011). Participants first completed 3 min of cycling with zero resistance, after which the work rate was increased at a rate of 10-15 W/min, depending on the individual's age-predicted maximum workload. They were asked to maintain a cadence of 55-60 rpm at all times and to continue cycling until they reached their limit of exercise tolerance; the test was stopped by the researchers if a patient desaturated to an oxygen level below $85 \%$. Throughout the test, which included 6-min periods of rest before and after exercise, a continuous Holter ECG was recorded (Pathfinder/Lifecard Digital CF system; Spacelabs Healthcare Ltd., Hertford, UK), providing ECG data with a 1,024-Hz sampling frequency, 12-bit resolution and a sample accuracy of 2.5 $\mu \mathrm{V}$. The ECG leads were positioned in the 'modified V5, CC5, modified V5R' electrode configuration. Patients were also asked to breathe through a facemask which allowed us to sample the air that they inspire/expire using an online respiratory gas analyser (MGC Ultima CardiO2; MGC Diagnostics, St Paul, MN, USA) and blood oxygen saturation was continuously monitored (Nonin Model 7500; Nonin Medical Inc., Plymouth, MN, USA).

Following baseline measurements participants began a 6-week PR programme (two sessions per week, $2 \mathrm{hr}$ per session). This programme consisted of 10 min of 'warming up' (20 sets of 'marching on the spot,' 'heal digs,' 'knee lifts,' 'shoulder rolls,' 'knee bends,' and 'high knees') followed by a range of strength training exercises ('bicep curls,' 'triceps extension,' 'dumbbell press,' 'dumbbell fly,' 'leg extension,' and 'dumbbell squats') and cardiovascular exercises (choice of treadmill, bicycle ergometer or cross-trainer exercise). Each patient was asked to choose a weight that they could safely handle at the beginning of the rehabilitation for use in each exercise, and they were encouraged to increase their weights on each day of rehabilitation as the programme progressed. For the cardiovascular exercise patients were asked to select a mode of exercise with which they were comfortable, which was maintained throughout the programme but with increasing workload (resistance) as rehabilitation progressed. The strength 
exercises lasted 5 min each and the cardiovascular exercise lasted $10 \mathrm{~min}$, followed by a 10-min cool-down period (which included sets of 'hip flexor stretch,' 'thigh stretch,' 'calf stretch,' and 'lower back stretch'). Upon completion of the rehabilitation programme patients were asked to return to the hospital for repeat physiological and functional assessments.

\section{Data analysis}

ECG recordings were assessed for quality by human observation using the Pathfinder system, primarily to verify the absence of excessive noise or artefact. Beat-to-beat cardiac intervals (RR) were then measured automatically by the Pathfinder system (using a proprietary algorithm) and any spurious data points (artefacts) were removed manually prior to subsequent analysis. The $\mathrm{RR}$ data were then analysed using the Kubios HRV software package (version 3.1) (Tarvainen et al., 2014) in three separate but contiguous sections covering the pre-exercise (rest), exercise, and recovery (postexercise) stages. The RR data within each of these sections was first detrended using the 'Smooth priors' function (Lambda $=500$ ). HR and $\mathrm{HRV}$ variables were next quantified according to the European Society of Cardiology Task Force guidelines (Task Force of the European Society of Cardiology and the North American Society of Pacing and Electrophysiology, 1996): (a) RMSSD (square root of the mean squared differences of successive RR intervals), (b) SDRR (standard deviation of RR intervals), (c) total power (TP; total amount of 'power' [variance] in the bandwidth $\sim 0$ to $0.4 \mathrm{~Hz}$ ), (d) VLF (very low frequency power, in the bandwidth $0.003-0.1 \mathrm{~Hz}$ ), (e) LF (low-frequency power in the bandwidth $0.04-0.15 \mathrm{~Hz}$ ), (f) $\mathrm{HF}$ (high-frequency power in the bandwidth $0.15-0.4 \mathrm{~Hz}$ ), (g) LFn (normalised low frequency power): $L F n=L F /(T P-V L F),(h)$ HFn (normalised high-frequency power): $\mathrm{HFn}=\mathrm{HF} /(\mathrm{TP}-\mathrm{VLF}$ ), and (i) the ratio $\mathrm{LF} / \mathrm{HF}$.

We used the PRSA procedure (Bauer et al., 2006) to quantify the acceleration capacity (AC) and deceleration capacity (DC): (a) multiple DC and AC 'anchor points' were identified, respectively, as those $\mathrm{RR}$ intervals longer than or shorter than the preceding $\mathrm{RR}$ interval; (b) a truncated RR time series was defined around each of the anchor points (a 61-point series, including 30 points preceding and following the anchor point); (c) the truncated $\mathrm{RR}$ time-series were aligned (separately for $\mathrm{DC}$ and $\mathrm{AC}$ anchor points) and averaged to form the PRSA time-series; (d) DC and AC were calculated as $\left[\left(\mathrm{X}_{0}+\mathrm{X}_{1}\right)-\left(\mathrm{X}_{-1}+\mathrm{X}_{-2}\right)\right] / 4$, where $\mathrm{X}_{0}$ and $\mathrm{X}_{1}$ are the averaged $\mathrm{RR}$ intervals at the anchor point and the immediately following point, and $\mathrm{X}_{-1}$ and $\mathrm{X}_{-2}$ are the two averaged $\mathrm{RR}$ intervals immediately preceding the anchor point. Group mean Accel- eration and Deceleration vectors (used for visualization) were calculated after standardising the individual RR time-series (to minimize interindividual differences in resting $\mathrm{RR}$ )-this was performed by subtracting the mean of the $12 \mathrm{RR}$ intervals immediately preceding interval $\mathrm{X}_{-2}$ from each $\mathrm{RR}$ value in the time-series.

The rates of oxygen uptake $\left(\mathrm{VO}_{2}\right)$ and carbon dioxide production $\left(\mathrm{VCO}_{2}\right)$ and minute ventilation (Ve) were measured from the breathby-breath respiratory gas data using the Breeze computer software (Breeze Software 6.4.1, Medical Graphics, St Paul, MN, USA). Forced expiratory volume in $1 \mathrm{sec}$ and forced vital capacity were measured using the spirometry test function of the online respiratory gas analyser. The maximum rate of oxygen uptake $\left(\mathrm{VO}_{2 \max }\right)$ was determined by averaging the $\mathrm{VO}_{2}$ values from the last $10 \mathrm{sec}$ before the limit of exercise was reached.

\section{Statistical analysis}

Matlab (version 2017b, The Mathworks, Cambridge, UK) was used to perform descriptive statistical analysis and to evaluative the nature of their distributions. The One-Sample KolomogorovSmirnov Test was used to test the null hypothesis that each groupwide HRV and PRSA-derived variable belonged to a standard normal distribution. If this assumption was violated then the influence of the rehabilitation intervention on each variable was assessed using Wilcoxon Signed Rank tests, and data were present-

Table 2. Six-minute walk distance (6MWD) and maximum rate of oxygen uptake $\left(\mathrm{VO}_{2 \max }\right)$ pre- and postpulmonary rehabilitation

\begin{tabular}{lccccc}
\hline \multirow{2}{*}{ Patient ID } & \multicolumn{2}{c}{$6 \mathrm{MWD}(\mathrm{m})$} & & \multicolumn{2}{c}{$\mathrm{VO}_{\text {2 } \max }(\mathrm{mL} / \mathrm{kg} / \mathrm{min})$} \\
\cline { 2 - 3 } \cline { 5 - 6 } & Pre-PR & Post-PR & & Pre-PR & Post-PR \\
\hline P01 & 370 & 362 & & 14.9 & 15.2 \\
P02 & 200 & 304 & & 10.0 & 8.4 \\
P03 & 290 & 430 & & 18.7 & 17.1 \\
P04 & 358 & 373 & & 13.1 & 12.8 \\
P05 & 181 & 300 & & 14.6 & 13.5 \\
P06 & 384 & 480 & & 16.0 & 12.5 \\
P07 & 407 & 427 & & 18.3 & 19.3 \\
P08 & 300 & 322 & & 15.1 & 9.6 \\
P09 & 222 & NA & & 14.3 & 12.8 \\
P10 & 457 & 440 & & 28.4 & 26.2 \\
P11 & NA & NA & & 12.5 & 15.4 \\
P12 & NA & NA & & 14.0 & 18.2 \\
P13 & 310 & 304 & & 9.5 & 16.9 \\
P14 & NA & NA & & 14.3 & 17.1 \\
P15 & 419 & NA & 13.9 & 15.3 \\
\hline
\end{tabular}

$\mathrm{PR}$, pulmonary rehabilitation; NA, not available. 
ed as median (M) and lower/upper quartile $(\mathrm{Q} 1, \mathrm{Q} 2)$ values. Onesample Kolmogorov-Smirnov tests showed that none of the quan- tified variables were normally-distributed. Consequently the Wilcoxon Signed-rank test was used to assess the influence of PR for

Table 3. Heart rate variability indices pre- and postpulmonary rehabilitation

\begin{tabular}{|c|c|c|c|c|c|c|c|c|c|c|c|c|c|}
\hline Variable & & & $\mathrm{HR}$ (bpm) & $\mathrm{RR}$ (msec) & SDNN (msec) & RMSSD (msec) & $\operatorname{VLF}\left(\mathrm{msec}^{2}\right)$ & $\operatorname{LF}\left(\mathrm{msec}^{2}\right)$ & $\mathrm{HF}\left(\mathrm{msec}^{2}\right)$ & $\operatorname{LFn}(\%)$ & $\mathrm{HFn}(\%)$ & LF/HF & $\mathrm{TP}\left(\mathrm{msec}^{2}\right)$ \\
\hline \multirow[t]{9}{*}{ Pre-PR } & Rest & M & 85.4 & 702.9 & 14.7 & 13.6 & 6.7 & 57.1 & 19.7 & 56.7 & 43.0 & 1.3 & 94.4 \\
\hline & & 01 & 74.3 & 586.0 & 8.4 & 11.8 & 3.1 & 10.6 & 11.9 & 41.4 & 30.4 & 0.7 & 35.2 \\
\hline & & 03 & 102.5 & 816.0 & 27.7 & 18.3 & 37.8 & 162.6 & 72.9 & 67.1 & 57.3 & 2.3 & 249.3 \\
\hline & Ex & M & 101.0 & 594.2 & 13.5 & 19.9 & 2.9 & 20.0 & 20.0 & 39.6 & 59.4 & 0.7 & 63.0 \\
\hline & & 01 & 84.2 & 536.4 & 9.4 & 13.3 & 1.2 & 6.6 & 7.6 & 27.5 & 39.4 & 0.4 & 22.0 \\
\hline & & 03 & 111.9 & 712.0 & 32.9 & 40.9 & 20.9 & 191.4 & 117.8 & 60.1 & 72.0 & 1.5 & 347.2 \\
\hline & $\operatorname{Rec}$ & M & 94.0 & 638.1 & 16.0 & 15.6 & 18.7 & 60.9 & 48.6 & 47.5 & 52.3 & 0.9 & 136.0 \\
\hline & & 01 & 86.5 & 548.0 & 11.2 & 11.1 & 5.9 & 34.1 & 10.5 & 33.1 & 15.8 & 0.5 & 50.3 \\
\hline & & 03 & 109.6 & 693.3 & 34.6 & 50.8 & 41.0 & 322.6 & 222.9 & 84.0 & 66.4 & 5.6 & 606.3 \\
\hline \multirow[t]{9}{*}{ Post-PR } & Rest & M & 86.6 & 692.7 & 15.0 & 23.1 & 9.3 & 72.4 & 53.2 & 61.6 & 38.2 & 1.6 & 153.9 \\
\hline & & 01 & 79.4 & 641.2 & 12.3 & 15.1 & 4.2 & 42.2 & 15.3 & 39.5 & 19.6 & 0.6 & 93.1 \\
\hline & & 03 & 93.6 & 755.3 & 20.6 & 31.9 & 42.2 & 147.9 & 123.4 & 80.2 & 59.6 & 4.1 & 210.4 \\
\hline & Ex & M & 112.5 & 549.4 & 23.6 & 34.9 & 7.1 & 45.1 & 73.8 & 36.3 & 62.3 & 0.5 & 119.6 \\
\hline & & 01 & 93.2 & 522.3 & 11.0 & 19.7 & 2.6 & 20.5 & 8.7 & 26.0 & 43.6 & 0.4 & 39.9 \\
\hline & & 03 & 115.4 & 638.8 & 31.3 & 43.6 & 13.9 & 110.4 & 316.8 & 56.3 & 73.4 & 1.3 & 527.1 \\
\hline & $\operatorname{Rec}$ & M & 110.9 & 541.2 & 16.5 & 26.2 & 5.3 & 60.5 & 43.2 & 34.4 & 64.9 & 0.5 & 143.1 \\
\hline & & 01 & 90.6 & 513.3 & 12.5 & 17.8 & 2.2 & 15.5 & 11.7 & 30.0 & 32.7 & 0.4 & 46.1 \\
\hline & & 03 & 116.9 & 665.9 & 30.0 & 43.3 & 12.2 & 146.4 & 173.2 & 67.1 & 69.2 & 2.2 & 349.0 \\
\hline
\end{tabular}

Median (M) and the lower (01) and upper (Q3) quartile values are shown, separately for the pre-exercise (Rest), exercise (Ex) and recover (Rec) periods.

HR, heart rate; RR, beat-to-beat cardiac intervals; SDNN, standard deviation of the normal-to-normal (sinus origin) cardiac intervals; RMSSD, square root of the mean squared differences of successive RR intervals; VLF, very low frequency; LF, low frequency; HF, high frequency; LFn, normalized low frequency; HFn, normalized high frequency; LF/HF, low frequency/high frequency; TP, total power; PR, pulmonary rehabilitation.

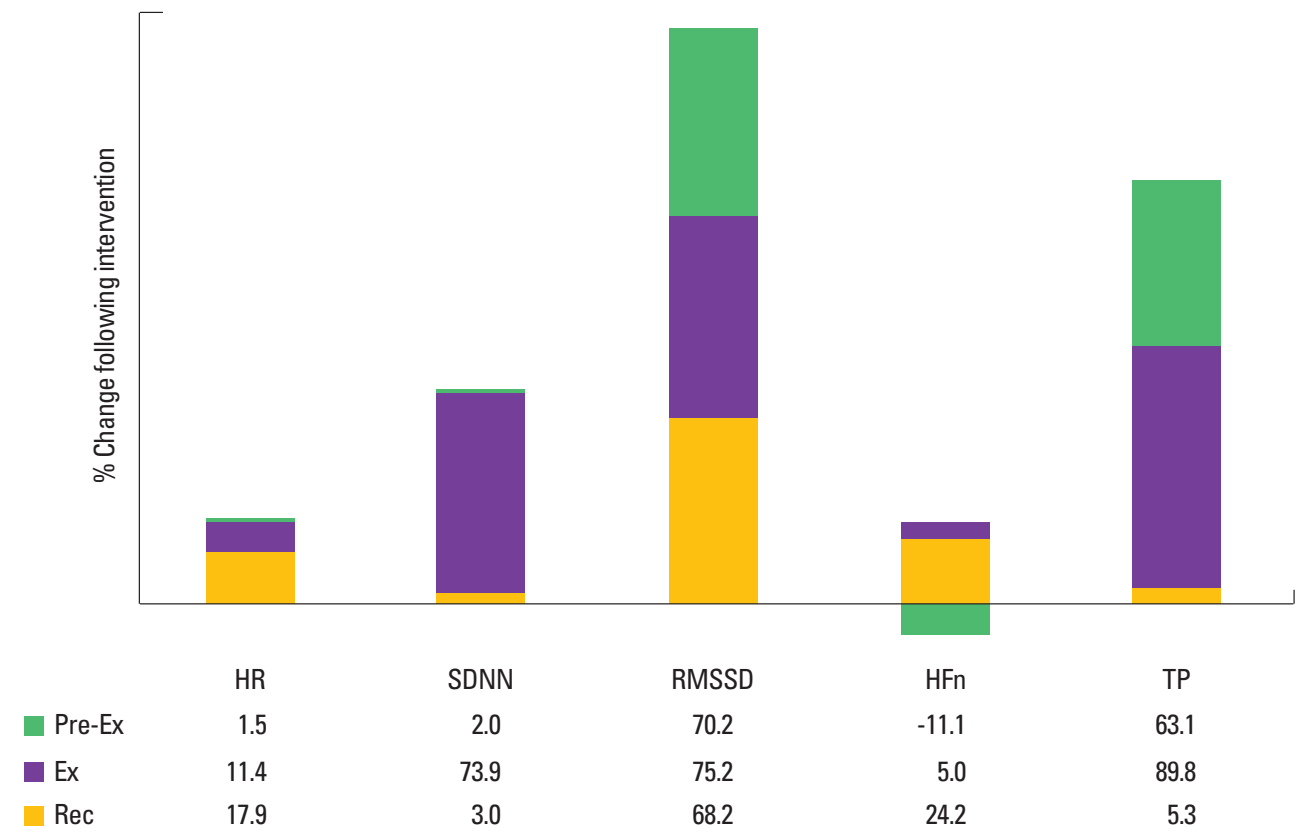

Fig. 1. Percentage change in heart rate variability indices following pulmonary rehabilitation. HR, heart rate; SDNN, standard deviation of the normal-to-normal (sinus origin) cardiac intervals; RMSSD, square root of the mean squared differences of successive RR intervals; HFn, normalized high frequency; TP, total power; Ex, exercise; Rec, recover. 
each variable. Statistical significance was assumed at the $P<0.05$ level.

\section{RESULTS}

Values for 6MWD and maximum rate of oxygen uptake before and after PR are shown in Table 2. For the group as a whole, 6MWT performance was poorer following $\mathrm{PR}(P=0.037, z=-2.09, \mathrm{ES}=$ 0.41 ) whilst $\mathrm{VO}_{2 \max }$ was unchanged $(P=0.93, z=0.09, \mathrm{ES}=0.02)$.
The HRV indices calculated pre- and postintervention for the pre-exercise (Rest), exercise (Ex), and recover (Rec) periods are shown in Table 3. This shows that the intervention did not influence any of the HRV variables during the Pre-Exercise $(P=0.14$ $0.99)$ or Exercise $(P=0.12-0.76)$ states. HR was greater (and RR was lower) postintervention during the Recovery state $(P=0.036$, $z=-2.10, \mathrm{ES}=0.38)$ and both $\operatorname{HFn}(P=0.069, z=-1.82, \mathrm{ES}=$ $0.33)$ and $\mathrm{LF} / \mathrm{HF}(P=0.047, z=1.99, \mathrm{ES}=0.36)$ showed trends towards greater high-frequency dominance during recovery
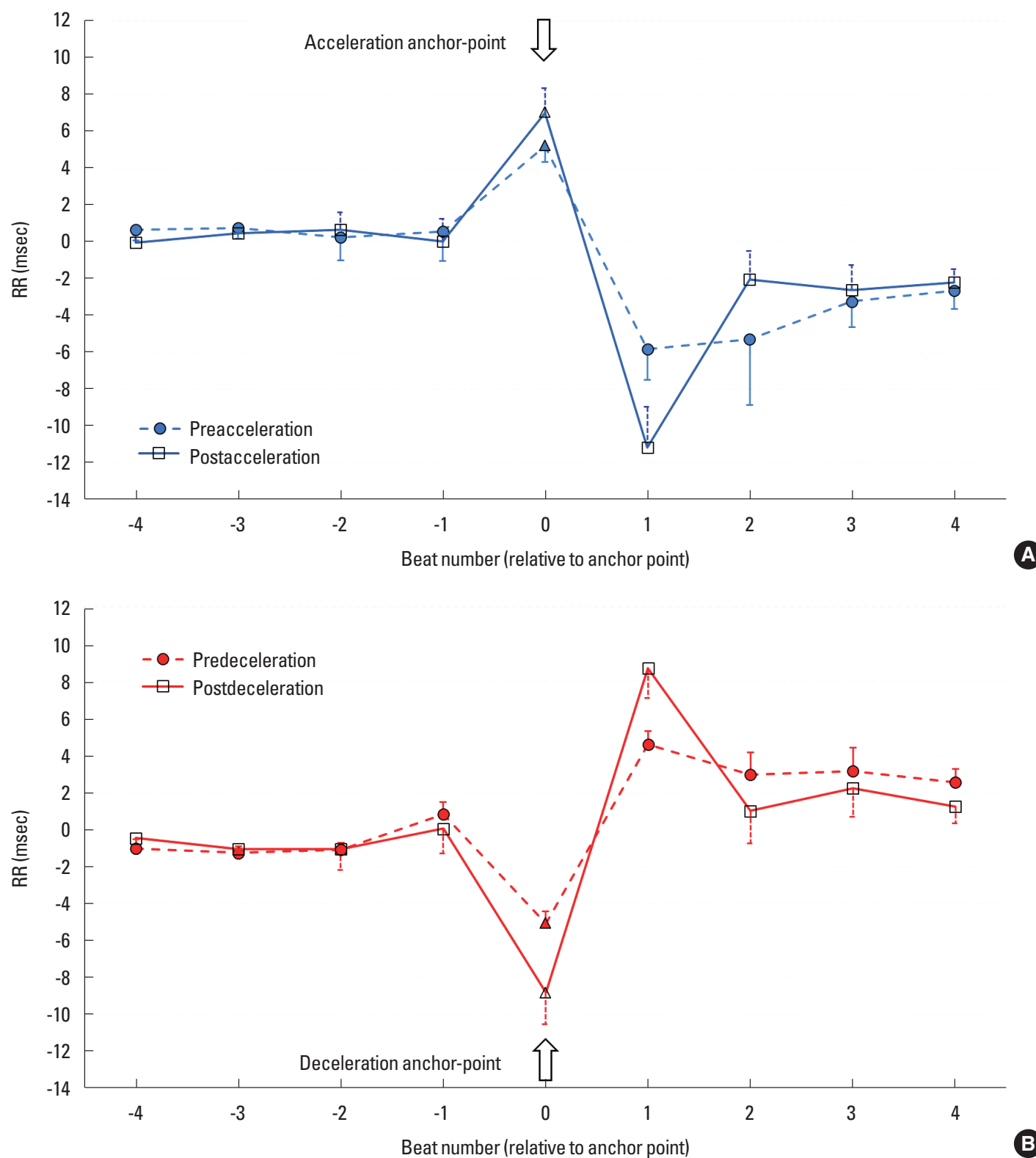

Fig. 2. Group mean ( \pm standard error of the mean) of the normalized RR time-series around the identified acceleration (A) and deceleration anchor-points (B). Individual participants' data were normalized by subtracting the mean of the $12 \mathrm{RR}$ intervals immediately preceding the anchor point. RR, beat-to-beat cardiac intervals. 


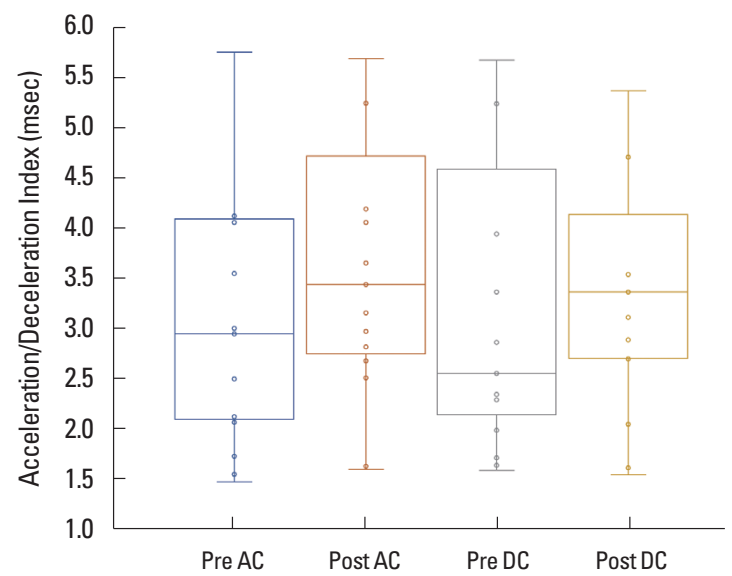

Fig. 3. Box-and-whisker plot of acceleration and deceleration indices pre and post pulmonary rehabilitation. Absolute values are shown for $\mathrm{AC}$; circles show individual values; horizontal lines in boxes indicate median, lower and upper quartile values; whiskers show range of values. $\mathrm{AC}$, acceleration capacity; $\mathrm{DC}$, deceleration capacity.

postintervention (other variables: $P=0.21-0.72$ ). The relative changes in HRV (HR, SDNN, RMSSD, LFn, and TP) following the intervention (expressed as percentages) are shown in Fig. 1 (the remaining variables are largely redundant for this purpose as they are closely related to these five variables).

To illustrate the PRSA averaging procedure, Fig. 2 shows the group mean ( \pm standard error of the mean) of the normalized $R R$ time-series around the identified acceleration and deceleration turning-points. Fig. 3 is a box-and-whisker plot of the $\mathrm{AC}$ and DC pre- and postintervention: the median (lower quartile, upper quartile) $\mathrm{AC}$ was $-2.7 \mathrm{msec}(-1.8,-5.3)$ preintervention and -3.2 msec $(-2.5,-4.9)$ postintervention; the DC was 2.3 msec $(1.8,4.8)$ preintervention and $3.2 \mathrm{msec}(2.5,4.3)$ postintervention. Wilcoxon Signed Rank tests revealed no influence of the intervention on either $\mathrm{AC}(P=0.53, z=0.63, \mathrm{ES}=0.11)$ or $\mathrm{DC}(P=0.73, z=-0.35$, $\mathrm{ES}=-0.06)$ indices.

\section{DISCUSSION}

We believe this is the first study to investigate the effect of a PR programme on HR responsiveness in people with UIP. We found that HR was elevated by 11\%-18\% during Exercise and Recovery states postrehabilitation, suggesting that participants' heart function (HR increase in response to exercise) was improved postrehabilitation. Statistically, HRV was not altered by the rehabilitation intervention during any of the three stages of our protocol (rest, exercise, recovery). However, we did observe substantial relative (post- vs. prerehabilitation) changes that are worthy of note: (a) a greater pre-exercise parasympathetic influence (RMSSD elevated by 70\%), (b) greater sympathetic and parasympathetic influences during Exercise (SDNN and RMSSD elevated by 75\%), and (c) a greater parasympathetic influence during postexercise (RMSSD elevated by 68\%, HFn elevated by $24 \%$ ). Whilst the numbers are small, we found that neither the acceleration index nor the deceleration index were statistically influenced by the intervention. However, we did observe trends for relative (postvs. prerehabilitation) improvements in these indices (changes of $19 \%$ and $38 \%$, respectively).

Our qualitative observations of altered HRV following physical rehabilitation suggest that this intervention had a positive influence on patients' cardiac autonomic regulation profiles. Conditions of stress (such as moderate physical exercise, a change in posture from supine to standing or mental processing tasks) alter the sympatho-vagal balance towards a more sympathetic-dominant state; this is reflected in frequency-domain HRV by a relative increase in LF power (reduction in HF power) and a reduction in $\mathrm{TP}$, with a corresponding relative increase in SDNN (reduction in RMSSD) in time-domain HRV (Task Force of the European Society of Cardiology and the North American Society of Pacing and Electrophysiology, 1996). At high work rates LF, HF and TP components of HRV are diminished compared with rest and lower work rates (Cottin et al., 2004; Lewis and Short, 2010). During progressive maximal exercise LF and HF are reduced exponentially (Perini et al., 2000; Tulppo et al., 1996) and Lewis et al. (2007) showed that the decay constants of HRV indices in each bandwidth are linearly related to maximal work rate. In the present study, the $70 \%$ postintervention increase in RMSSD during rest suggests that patients' resting cardiac autonomic states became more typical of the relaxed (parasympathetic-dominant) states displayed in healthy people. Increases of similar magnitude (75\%) in RMSSD and SDNN during exercise indicate that patients were working at lower relative intensities following the intervention (they found the same activity easier to perform so the exponential-reduction in HRV power was not as great). Finally, following the intervention patients displayed a large (68\%) increase in RMSSD during recovery, and this faster return to the pre-exercise (parasympathetic-dominant) state likely reflects improved cardiac 'fitness'.

We previously observed significantly reduced values for $\mathrm{AC}$ and $D C$ in older compared with younger healthy individuals: older (mean age 66 years; $A C=-5$ to $-7 \mathrm{msec} ; \mathrm{DC}=4.5$ to $6.5 \mathrm{msec}$ ) and younger (mean age 21 years; $A C=-10$ to $-12 ; D C=8$ to 10 msec) (Lewis et al., 2011). In that study patients with COPD had 
qualitatively reduced values (AC diminished by 4-6 msec; DC diminished by $2-3 \mathrm{msec}$ ) compared with similarly aged healthy people. A further study by our group added to these data, showing that $\mathrm{AC}$ and $\mathrm{DC}$ are reduced in middle-aged (mean 45 years) compared with younger (mean 24 years) healthy individuals (mean values $\mathrm{AC}=-8.4 /-10.3$ and $\mathrm{DC}=7.6 / 9.5 \mathrm{msec}$, respectively) and that both $A C$ and DC are enhanced in aerobically superior individuals (McNarry and Lewis, 2012). The AC and DC values observed for patients with IPF in the present study ( $A C=-2.7$ to $-3.2 \mathrm{msec} ; \mathrm{DC}=2.3$ to $3.2 \mathrm{msec}$ ) are around $50 \%$ lower than those of similarly aged healthy people and are similar to those of individuals with COPD who have not received oxygen therapy. Visualisation of the RR time-series around the identified acceleration and deceleration anchor-points enabled the following observations: (a) acceleration anchor-points occurred at larger $R R$ values after rehabilitation, (b) deceleration anchor-points occurred at smaller RR values after rehabilitation, (c) immediately post-anchor acceleration was greater ( $R R$ values achieved were smaller) after rehabilitation, and (d) immediate post-anchor deceleration was greater ( $\mathrm{RR}$ values achieved were larger) after rehabilitation. These observations mean that, following rehabilitation: (a) HR was allowed to reach lower values before triggering a larger tachycardic response and (b) HR was allowed to reach higher values before triggering a larger bradycardic response. We suggest that these results provide evidence that the physical rehabilitation intervention may have improved the dynamic range of HR responsiveness in our patients with UIP. We further speculate that this would have enhanced patients' physical function during everyday tasks (being mindful that the $\mathrm{AC}$ and $\mathrm{DC}$ indices were calculated as an ensemble across each of the three physiological states in our protocol).

The diminished cardiac acceleration and deceleration capacities in UIP patients reflect the autonomic dysfunction that is also indicated qualitatively by the HRV variables for this group of individuals. Postintervention improvements in AC and DC indices appear to reflect the observed shift towards 'healthy' HRV values for these patients. Taken together, these changes suggest that physical rehabilitation had a positive 'normalising' influence on cardiac autonomic modulation of HR in people with UIP.

When considering the results reported here we must be mindful of potential confounders relating to both the UIP patient population and also our study design. Whilst we consider our sample of 15 patients to be representative of people with UIP, one limitation of this study is the relatively small number of participants, which reduced the statistical power of our analyses. We considered the influence of potential confounders on the ANS and thus on the measured parameters. We therefore excluded patients who had comorbid conditions that might influence cardiac or ANS function. However, we were also mindful of the potential influence of too strict a set of exclusion criteria on both recruitment and the applicability of our results to a wider population or people with UIP. With this in mind we took the decision to not exclude individuals with controlled diabetes from the study: five (27\%) of our patient cohort were taking medication to treat type II diabetes. Furthermore, patients acted as their own controls and care was taken to ensure that the only change between the monitoring days was the intervening rehabilitation intervention.

\section{CONFLICT OF INTEREST}

No potential conflict of interest relevant to this article was reported.

\section{ACKNOWLEDGMENTS}

We would like to express our thanks for the support of Caroline Evered, Adrian Paton, Helen Davies, Marie Clare Oliver (Pulmonary Rehabilitation Team, ABMU Health Board), who conducted the rehabilitation programme; Simon Hilldrup (Respiratory Unit, ABMU Health Board), who provided advice and assistance with regard to respiratory data collection; Dr. Melitta McNarry (Swansea University) for contributing to study design.

\section{REFERENCES}

American Thoracic Society. Idiopathic pulmonary fibrosis: diagnosis and treatment. International consensus statement. American Thoracic Society (ATS), and the European Respiratory Society (ERS). Am J Respir Crit Care Med 2000;161(2 Pt 1):646-664.

Arena R, Sietsema KE. Cardiopulmonary exercise testing in the clinical evaluation of patients with heart and lung disease. Circulation 2011; 123:668-680.

ATS Committee on Proficiency Standards for Clinical Pulmonary Function Laboratories. ATS statement: guidelines for the six-minute walk test. Am J Respir Crit Care Med 2002;166:111-117.

Bartels MN, Jelic S, Ngai P, Basner RC, DeMeersman RE. High-frequency modulation of heart rate variability during exercise in patients with COPD. Chest 2003;124:863-869.

Bauer A, Kantelhardt JW, Bunde A, Barthel P, Schneider R, Malik M, Schmidt $\mathrm{G}$. Phase-rectified signal averaging detects quasi-periodicities 
in non-stationary data. Phys A: Stat Mech Appl 2006;364:423-434.

Bolton CE, Bevan-Smith EF, Blakey JD, Crowe P, Elkin SL, Garrod R, Greening NJ, Heslop K, Hull JH, Man WD, Morgan MD, Proud D, Roberts CM, Sewell L, Singh SJ, Walker PP, Walmsley S; British Thoracic Society Pulmonary Rehabilitation Guideline Development Group; British Thoracic Society Standards of Care Committee. British Thoracic Society guideline on pulmonary rehabilitation in adults. Thorax 2013;68 Suppl 2:ii1-30.

Cottin F, Médigue C, Leprêtre PM, Papelier Y, Koralsztein JP, Billat V. Heart rate variability during exercise performed below and above ventilatory threshold. Med Sci Sports Exerc 2004;36:594-600.

Dowman LM, McDonald CF, Hill CJ, Lee AL, Barker K, Boote C, Glaspole I, Goh NSL, Southcott AM, Burge AT, Gillies R, Martin A, Holland $\mathrm{AE}$. The evidence of benefits of exercise training in interstitial lung disease: a randomised controlled trial. Thorax 2017;72:610-619.

Gross TJ, Hunninghake GW. Idiopathic pulmonary fibrosis. N Engl J Med 2001;345:517-525.

Kelly CA, Saravanan V, Nisar M, Arthanari S, Woodhead FA, Price-Forbes AN, Dawson J, Sathi N, Ahmad Y, Koduri G, Young A; British Rheumatoid Interstitial Lung (BRILL) Network. Rheumatoid arthritis-related interstitial lung disease: associations, prognostic factors and physiological and radiological characteristics - a large multicentre UK study. Rheumatology (Oxford) 2014;53:1676-1682.

Lewis MJ, Annandale J, D'Silva LA, Davies RE, Reed Z, Lewis KE. Influence of long-term oxygen therapy on cardiac acceleration and deceleration capacity in hypoxic patients with chronic obstructive pulmonary disease. Clin Physiol Funct Imaging 2011;31:258-265.

Lewis MJ, Annandale J, Lewis KE. Influence of long-term oxygen therapy on heart rate and QT time-series in hypoxic patients with chronic obstructive pulmonary disease. Clin Physiol Funct Imaging 2009;29:431439.

Lewis MJ, Kingsley M, Short AL, Simpson K. Rate of reduction of heart rate variability during exercise as an index of physical work capacity. Scand J Med Sci Sports 2007;17:696-702.

Lewis MJ, Short AL. Exercise and cardiac regulation: what can electrocardiographic time series tell us? Scand J Med Sci Sports 2010;20:794-804.

Ley B, Collard HR, King TE Jr. Clinical course and prediction of survival in idiopathic pulmonary fibrosis. Am J Respir Crit Care Med 2011;183: 431-440.

MacPhee SL, Shoemaker JK, Paterson DH, Kowalchuk JM. Kinetics of $\mathrm{O}_{2}$ uptake, leg blood flow, and muscle deoxygenation are slowed in the upper compared with lower region of the moderate-intensity exercise domain. J Appl Physiol (1985) 2005;99:1822-1834.

McNarry MA, Harrison NK, Withers T, Chinnappa N, Lewis MJ. Pulmonary oxygen uptake and muscle deoxygenation kinetics during heavy intensity cycling exercise in patients with emphysema and idiopathic pulmonary fibrosis. BMC Pulm Med 2017;17:26.

McNarry MA, Lewis MJ. Interaction between age and aerobic fitness in determining heart rate dynamics. Physiol Meas 2012;33:901-914.

Perini R, Milesi S, Fisher NM, Pendergast DR, Veicsteinas A. Heart rate variability during dynamic exercise in elderly males and females. Eur J Appl Physiol 2000;82:8-15.

Raghu G, Collard HR, Egan JJ, Martinez FJ, Behr J, Brown KK, Colby TV, Cordier JF, Flaherty KR, Lasky JA, Lynch DA, Ryu JH, Swigris JJ, Wells AU, Ancochea J, Bouros D, Carvalho C, Costabel U, Ebina M, Hansell DM, Johkoh T, Kim DS, King TE Jr, Kondoh Y, Myers J, Müller NL, Nicholson AG, Richeldi L, Selman M, Dudden RF, Griss BS, Protzko SL, Schünemann HJ; ATS/ERS/JRS/ALAT Committee on Idiopathic Pulmonary Fibrosis. An official ATS/ERS/JRS/ALAT statement: idiopathic pulmonary fibrosis: evidence-based guidelines for diagnosis and management. Am J Respir Crit Care Med 2011;183:788-824.

Raghu G, Rochwerg B, Zhang Y, Garcia CA, Azuma A, Behr J, Brozek JL, Collard HR, Cunningham W, Homma S, Johkoh T, Martinez FJ, Myers J, Protzko SL, Richeldi L, Rind D, Selman M, Theodore A, Wells AU, Hoogsteden H, Schünemann HJ; American Thoracic Society; European Respiratory Society; Japanese Respiratory Society; Latin American Thoracic Association. An official ATS/ERS/JRS/ALAT clinical practice guideline: Treatment of idiopathic pulmonary fibrosis. An update of the 2011 clinical practice guideline. Am J Respir Crit Care Med 2015;192:e3-19.

Spruit MA, Singh SJ, Garvey C, ZuWallack R, Nici L, Rochester C, Hill K, Holland AE, Lareau SC, Man WD, Pitta F, Sewell L, Raskin J, Bourbeau J, Crouch R, Franssen FM, Casaburi R, Vercoulen JH, Vogiatzis I, Gosselink R, Clini EM, Effing TW, Maltais F, van der Palen J, Troosters T, Janssen DJ, Collins E, Garcia-Aymerich J, Brooks D, Fahy BF, Puhan MA, Hoogendoorn M, Garrod R, Schols AM, Carlin B, Benzo R, Meek P, Morgan M, Rutten-van Mölken MP, Ries AL, Make B, Goldstein RS, Dowson CA, Brozek JL, Donner CF, Wouters EF; ATS/ERS Task Force on Pulmonary Rehabilitation. An official American Thoracic Society/European Respiratory Society statement: key concepts and advances in pulmonary rehabilitation. Am J Respir Crit Care Med 2013; 188:e13-64.

Tarvainen MP, Niskanen JP, Lipponen JA, Ranta-Aho PO, Karjalainen PA. Kubios HRV--heart rate variability analysis software. Comput Methods Programs Biomed 2014;113:210-220.

Task Force of the European Society of Cardiology and the North American Society of Pacing and Electrophysiology. Heart rate variability: standards of measurement, physiological interpretation and clinical use. Circulation 1996;93:1043-1065.

Tonelli R, Cocconcelli E, Lanini B, Romagnoli I, Florini F, Castaniere I, 
Andrisani D, Cerri S, Luppi F, Fantini R, Marchioni A, Beghè B, Gigliotti F, Clini EM. Effectiveness of pulmonary rehabilitation in patients with interstitial lung disease of different etiology: a multicenter prospective study. BMC Pulm Med 2017;17:130.

Tulppo MP, Mäkikallio TH, Takala TE, Seppänen T, Huikuri HV. Quanti- tative beat-to-beat analysis of heart rate dynamics during exercise. Am J Physiol 1996;271(1 Pt 2):H244-252.

Yoshida T, Whipp BJ. Dynamic asymmetries of cardiac output transients in response to muscular exercise in man. J Physiol 1994;480(Pt 2):355359. 